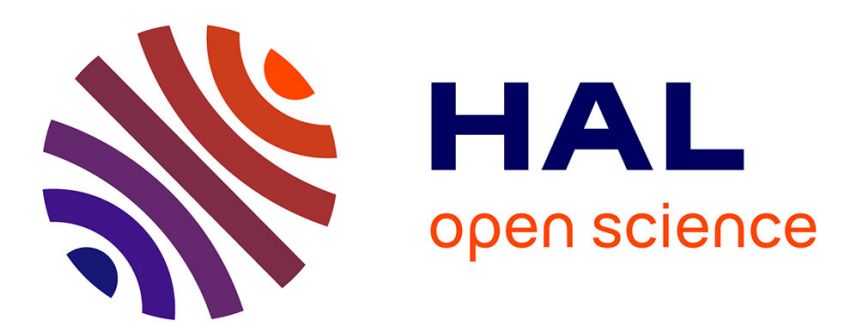

\title{
Conception of New Phase Dislocation-Based Nucleation at Reconstructive Martensitic Transformations
}

\author{
V. Letuchev, V. Vereshchagin, I. Alexina, M. Kashchenko
}

\section{To cite this version:}

V. Letuchev, V. Vereshchagin, I. Alexina, M. Kashchenko. Conception of New Phase DislocationBased Nucleation at Reconstructive Martensitic Transformations. Journal de Physique IV Proceedings, 1995, 05 (C8), pp.C8-151-C8-156. 10.1051/jp4:1995818 . jpa-00254067

\section{HAL Id: jpa-00254067 https://hal.science/jpa-00254067}

Submitted on 1 Jan 1995

HAL is a multi-disciplinary open access archive for the deposit and dissemination of scientific research documents, whether they are published or not. The documents may come from teaching and research institutions in France or abroad, or from public or private research centers.
L'archive ouverte pluridisciplinaire HAL, est destinée au dépôt et à la diffusion de documents scientifiques de niveau recherche, publiés ou non, émanant des établissements d'enseignement et de recherche français ou étrangers, des laboratoires publics ou privés. 


\title{
Conception of New Phase Dislocation-Based Nucleation at Reconstructive Martensitic Transformations
}

\author{
V.V. Letuchev, V.P. Vereshchagin, I.V. Alexina and M.P. Kashchenko \\ Ural State Wood Technology Academy, Physics Chair, Sybirskyi Trak 37, 620032, Ekaterinburg, Russia
}

\begin{abstract}
The role of dislocations and the dynamical mechanism controlling the structural reconstruction in the process of nucleation and wave growth of new phase unit crystals at martensitic transformations in metallic systems are discussed. It has been established that near some rectilinear dislocations with lines and Burgers's vectors typical for the original phase, there are areas where an elastically deformed state is characterized by package of particularities unambiguously corresponding to the well definite morphological attribute set (habit, orientation relationship, macroshear) of the martensite crystal. The distinctiveness of these areas for martensite nucleation is caused by character of strains reducing the magnitude of interphase energetic barrier. The elastic model of the dislocation-based nucleation center of martensitic crystal, allowing to select the dislocations being the most probable for nucleation and to make a martensitic crystal with the morphological attribute specific collection corresponding to each a dislocation, has been proposed. Such dislocations for certain $\mathrm{Fe}$-, $\mathrm{Cu}$ - and TiNi-based alloys are indicated. New results for titanium nickel are presented in more detail.
\end{abstract}

\section{INTRODUCTION}

Martensitic transformation (MT) is one of the most interesting variants of structural phase transitions in solids. A feature peculiar to martensitic transformations (MT) is a mechanism controlling a co-operative displacement of atoms. While on the subject of MT, we shall restrict our consideration to the transformation featuring the first-order phase transition attributes. For a long time the physical nature of the controlling mechanism remained incomprehensible, at least in substantial detail. The crystallogeometric theory predominated to treat the observed morphological features of MT [1]. This theory is a simple variant of the thermodynamical theory. It postulates mainly that the interphase contact along the invariant plane is profitable. The contact along such plane must actually minimize the energy of elastic distortions. The problem of theory consisted in describing the observed macroscopic morphological attributes of martensite: the habit plane (HP), the macroshear $\mathbf{S}$, orientation relationships between lattices of phases. The habit plane therewith was treated as a macroscopically invariant plane. Both a symmetry and lattice parameters of original and final phases was considered to be known. The homogeneous deformation was given in such a way as to ensure the required reconstruction of the unit cell. The additional inhomogeneous deformation was so matched that the main postulate was fulfilled. It is the last stage that was a creative part of the theoretician's work. It was limited to looking for suitable combination of twinning and shear. In spite of some failures the indicated approach decided the problem to be sought well. In the same time the sequential dynamical theory of nucleation and growth of martensite had not been worked out. But, evidently, the above-mentioned postulate could be expected to find its place in the construction of a new approach. 


\section{TREATMENT OF MORPHOLOGICAL ATTRIBUTES OF MARTENSITE IN THE FRAMEWORK OF CONTROLLING MECHANISM CONCEPTION}

Let us to move from the universal physical conception of short-range interaction. Then, it should be admitted that there is a mechanism controlling the displacement of atoms and ensuring the appearance of contact along an invariant plane (IP). The condition of the HP existence, it will be remembered, is:

$$
\varepsilon_{1}>0, \quad \varepsilon_{2}<0, \quad \varepsilon_{3}=0
$$

where $\varepsilon_{\mathrm{i}}$ - eigenvalues of deformation tensor $\varepsilon_{\mathrm{i}, \mathrm{j}}$.

The fulfillment of condition (1) causes an existence of two invariant planes and their normals $N_{I P}$ are expressed by the eigenvalues $\varepsilon_{1,2}$ and eigenvectors $\vec{\xi}_{1,2}$ of tensor $\varepsilon_{i, j}$ :

$$
\left(N_{\mathrm{IP}}\right)_{1,2} \| \vec{\xi}_{2} \pm k \vec{\xi}_{1}, \quad k=\frac{1-\left|\varepsilon_{2}\right|}{1+\varepsilon_{1} \mid} \cdot \sqrt{\frac{\varepsilon_{1}\left(2+\varepsilon_{1}\right)}{\left|\varepsilon_{2}\right|\left(2-\left|\varepsilon_{2}\right|\right)}}, \quad \vec{\xi}_{1} \perp \vec{\xi}_{2}, \quad\left|\vec{\xi}_{1,2}\right|=1 .
$$

The condition (1) shows that the controlling mechanism is connected with a space translation of plane deformation with tension and compression in two mutually orthogonal directions.

Because velocity of martensitic crystal growth is of the order of the sound one, the controlling mechanism is indubitably characterized by a wave nature. Two plane longitudinal waves running in orthogonal directions $\mathbf{n}_{1}$ and $\mathbf{n}_{2}$ represent the simplest model of such mechanism $[2,3]$. Indeed, superposition of waves results always to the appearance of area in the form of elongated rectangular parallelepiped where the conditions (1) are carried out. The transverse dimensions of parallelepiped are appropriate to one-half wavelengths and the orientation of its long axis is given by the vector product of wave normals $\mathbf{n}_{1}$ and $\mathbf{n}_{2}$. The line of intersection of the two wave fronts draws the plane (the wave habit) featuring the normal $\mathbf{N}_{w}$ :

$$
\left(\mathbf{N}_{w}\right)_{1,2} \| \mathbf{n}_{2} \pm \frac{c_{2}}{c_{1}} n_{1}, \quad\left|n_{1,2}\right|=1
$$

where $c_{1}$ and $c_{2}$ are the absolute values of vectors of wave velocities $c_{1,2}=c_{1,2} n_{1,2}$.

There is, however, a question about the manner in which the waves running along the well defined directions $n_{1,2}$ come into existence. From where does the crystal know about the plane which the new phase will be matched on? The answer is quite simple. Recall that we are dealing with a first-order structural transition in solids. In this case, the nucleation process is most likely to be heterogeneous. Consequently, there is a lattice defect disturbing the symmetry of parent phase. It is the defect creating the deformation close to the conditions (1) that highlights a plane profitable for the interphase contact. It must be emphasized that the highlighting of indicated plane is realized in a wave operation. For example, conditions

$$
n_{1}=\vec{\xi}_{1}, \quad n_{2}=\vec{\xi}_{2}, \quad k=\frac{c_{2}}{c_{1}}
$$

result to collinearity of the vectors $N_{I P}$ and $N_{w}$. Equalities $\mathbf{n}_{1,2}=\vec{\xi}_{1,2}$ suggest that a nucleation is accompanied by excitation of waves with normals oriented along the eigenvectors of the deformation tensor. This is quite reasonable because it is these directions that are highlighted. Thus, the problem to define the future habit reduces to the calculation of elastic fields of defects and wave velocities. The problem solution needs only the elastic moduli $C_{i, j}$ of original lattice near the temperature $M_{s}$ of the MT start.

Such formulation of the problem reveals the physical nature of controlling process. It allows incidently to identify the defects which play the role of nucleation centers of specific martensitic crystals. For many of the alloys with a cubic original lattice these defects as shown by calculations are the rectilinear dislocations. A number of aspects of the influence of dislocations on the nucleation was respected in the recent overview [4]. The specificity of rectilinear dislocations is explainable because ones are characterized by a slowly descending 
elastic field and consequentially they deform effectively the lattice and lower the interphase energetical barrier.

The dislocation line $\Lambda$ is invariant and the slip plane undergoes the smallest rotation (in comparison with another similar slip planes) in its proper elastic field. Because of this, it is these two attributes of dislocation that must appear in an orientation relationship between lattices of original and final phases. Such natural orientation relation is genetically connected with characteristics of the dislocation-based nucleation center (DNC).

The relationship (2) is also employed to calculate normals of weakly distorted planes (WDP). It should be noted that the conditions (4) can be fulfilled for WDP. If this is the case it is the WDP that will play the role of HP. We can take, as the measure of distortion, the parameter $\eta$ :

$$
\eta=\frac{\left|\varepsilon_{3}\right|}{\sqrt{\varepsilon_{1} \cdot\left|\varepsilon_{2}\right|}}
$$

It is easily seen that $\eta$ becomes equal to zero for the HP. In the case of the DNC the quantities $\varepsilon_{i}$ and the vectors $\vec{\xi}_{\mathrm{i}}$ in conditions (1), (2) are considered as functions of polar angle $\theta$. This angle lies in the plane being perpendicular to the dislocation line and is measured from the slip plane of dislocation. The question about the direction of macroshear $\mathbf{S}$ is solved in parallel with the choice of one of two orientations of $\mathbf{N}$ in the condition (2). For this purpose, the distortion tensor $\chi$ of elastic field of the DNC is presented as the sum of dyads:

$$
\chi=\mathbf{S}_{1} \cdot \mathbf{H}_{1}+\mathbf{S}_{2} \cdot \mathbf{H}_{2}+\mathbf{S}_{3} \cdot \vec{\xi}_{3} \text {, where } \mathbf{H}_{1}=\frac{\mathbf{N}_{1}}{\left|\mathbf{N}_{1}\right|}, \mathbf{H}_{2}=\frac{\mathbf{N}_{2}}{\left|\mathbf{N}_{2}\right|},\left|\vec{\xi}_{3}\right|=1 \text {. }
$$

In the area of angles $\theta$ profitable for nucleation one of vectors $\left(\mathbf{S}_{1}\right.$ or $\left.\mathbf{S}_{2}\right)$ is more by an order of magnitude than two another ones: $\left|\mathbf{S}_{1}\right| \gg\left|\mathbf{S}_{2,3}\right|$ or $\left|\mathbf{S}_{2}\right| \gg\left|\mathbf{S}_{1,3}\right|$. It is the characteristics of the main dyad in equation (6) that specify the expected directions of vectors $\mathbf{S}$ and $\mathbf{N}$.

\section{THE MOST PROBABLE DISLOCATION-BASED NUCLEATION CENTERS FOR CONCRETE SYSTEMS}

The results of similar analysis have already allowed to identify unambiguously the DNC for the $\gamma-\alpha$ MT in Fe-based alloys [2]. The dislocations exhibiting lines $\Lambda \|<1 \overline{1} 0\rangle_{\gamma}$, slip planes $\{111\}_{\gamma}$ and Burgers's vectors $b \|<10 \overline{1}>\gamma\left(60^{\circ}\right.$-dislocations) are the DNC for the HP $\{\tilde{5} 57\}_{\gamma}$ and $\{\tilde{2} 25\}_{\gamma}$. The sign " $\sim$ " marks the greatest index among two approximately equal ones $(\tilde{5}>$ $5, \tilde{2}>2$ ). On the other hand, the dislocations featuring $\Lambda \|<1 \overline{2} 1>_{\gamma}$, slip planes $\{111\}_{\gamma}$ and

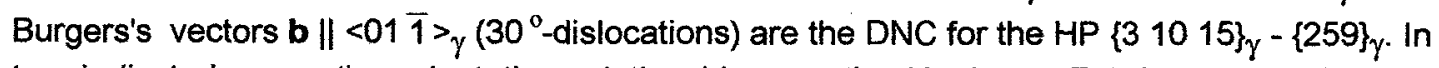
two indicated cases the orientation relationships are the Kurdumov-Zaks's and Nishiyama's ones accordingly.

The role of the DNC for both the bcc-fct transformation in the $\mathrm{Cu}-42 w \% \mathrm{Zn}$ and the reversed $\alpha$ $-\gamma$ MT in Fe-based alloys is played by dislocations with $\Lambda \|\langle 1 \overline{1} 1\rangle_{\alpha}$, slip plane $\{110\}_{\alpha}$. They result the HP of the type $\{561\}_{\alpha}$. The vector $\mathbf{b} \|\langle\overline{1} 11\rangle_{\alpha}$ is preferable. But this Burgers's vector can be chosen with confidence for the original state ordered in conformity with B2-type only; otherwise the vector $b$ can take the different orientations [5].

The transition $\mathrm{B} 2-\mathrm{B} 19$ in the alloy $\mathrm{Ti}-38$ at\% Ni -10 at\% Cu-2at\%Fe can result to appearance of crystals exhibiting the HP of type $\{334\}_{B 2}$. The indicated habit correlates with DNC featuring $\Lambda \|<1 \overline{1}_{0}>_{B 2}$, the slip plane $\{110\}_{B 2}$ and $b||<001>_{B 2}$ (edge dislocation) [6].

Let us next consider the transition $\mathrm{B} 2-\mathrm{B} 19$ in titanium nickel in more detail. This transformation can be related with the DNC of the following form: $\Lambda \|<1 \overline{2} 0>_{B 2}$, the slip plane $\{211\}_{B 2}$, the 
Burgers's vector $\mathbf{b} \|\langle 1 \overline{1} 1\rangle_{\mathrm{B} 2}$. It is interesting to note that in this situation one DNC can be associated with two habits of B19-crystals. According to [7], the thermally formed martensite takes the HP $\{0.780 .390 .48\}_{B 2}$ and the stress-induced one $-\{0.870 .410 .27\}_{B 2}$. Three sets of elastic moduli were used for analysis of elastic field of the DNC:

$$
\begin{array}{llll}
\mathrm{C}_{\mathrm{L}}=182.5 \mathrm{GPa}, & \mathrm{C}_{44}=33 \mathrm{GPa}, & C^{\prime}=16.5 \mathrm{GPa}, & A=2.0 \\
\mathrm{C}_{\mathrm{L}}=175.6 \mathrm{GPa}, & C_{44}=27 \mathrm{GPa}, & C^{\prime}=15.7 \mathrm{GPa}, & \AA \cong 1.7 \\
\mathrm{C}_{\mathrm{L}}=130.0 \mathrm{GPa}, & C_{44}=27 \mathrm{GPa}, & C^{\prime}=20.8 \mathrm{GPa}, & \dot{A} \cong 1.3
\end{array}
$$

where $A=C_{44} / C^{\prime}$. The collection (7) is taken from [8], (8) - from [9] for the temperature close to the temperature of start of B2-R transition. The collection (9) is a model and fits to the smaller anisotropy factor. The angle dependence of parameter $\eta$ for different sets of elastic moduluses is presented in the Figure 1. The HP of thermally formed martensite corresponds to the angle $\theta$

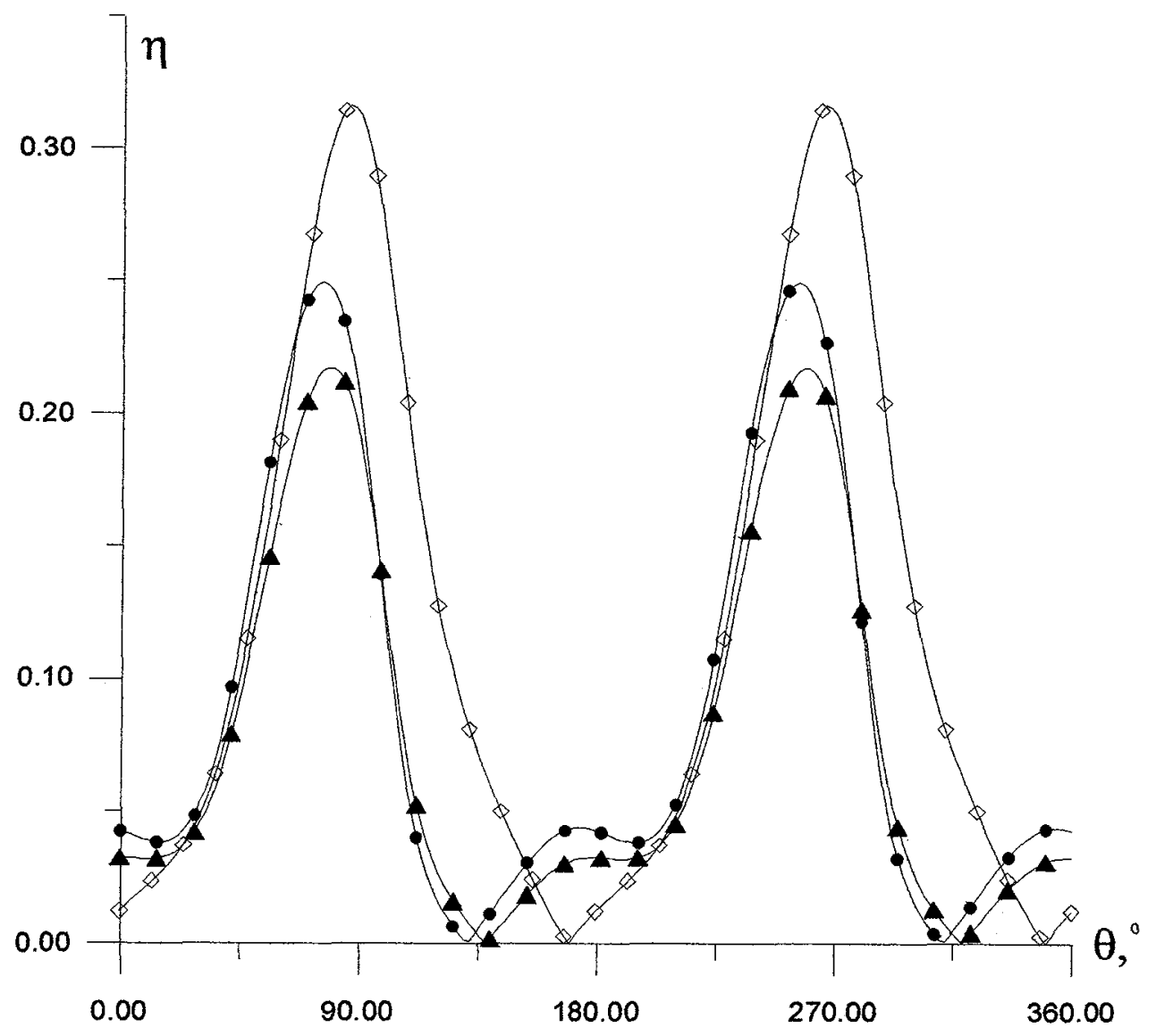

Figure 1. Parameter $\eta$ as a function of angle $\theta$. The sign corresponds to the anisotropy factor $A=2.0$, $\Delta-A=1.7$ and $\diamond-A=1.3$.

about $185^{\circ}$. It is the angle that correlates with one of minimums of parameter $\eta=\eta_{\min } \neq 0$ when the anisotropy factor $A=1.7$. In this case, in addition, the practically total coincidence between 
$N_{W D P}$ and $N_{W}$ takes place. The second minimum $\eta=0$ near the angle $\theta$ about $138^{\circ}$ is in line with the invariant plane but there is a significant discrepancy between the normals $\mathbf{N}_{\text {IP }}$ and $\mathrm{NW}_{\mathrm{W}}$ for such value of $\theta$. Nevertheless, there is the combination of moduli (9) which results to disappearance of minimum related with the weakly distorted plane near $\theta=190^{\circ}$; but, at the same time, the minimum $\eta$ corresponding to the invariant plane is displaced to the angle $\theta$ about $170^{\circ}$. It is the angle value that correlates with the thermally formed martensite habit plane satisfying the wave model. The Fugure 2 represents results of calculations of elastic energy of the DNC in the original state. It shows that there is the energy minimum near $\theta=170^{\circ}$.

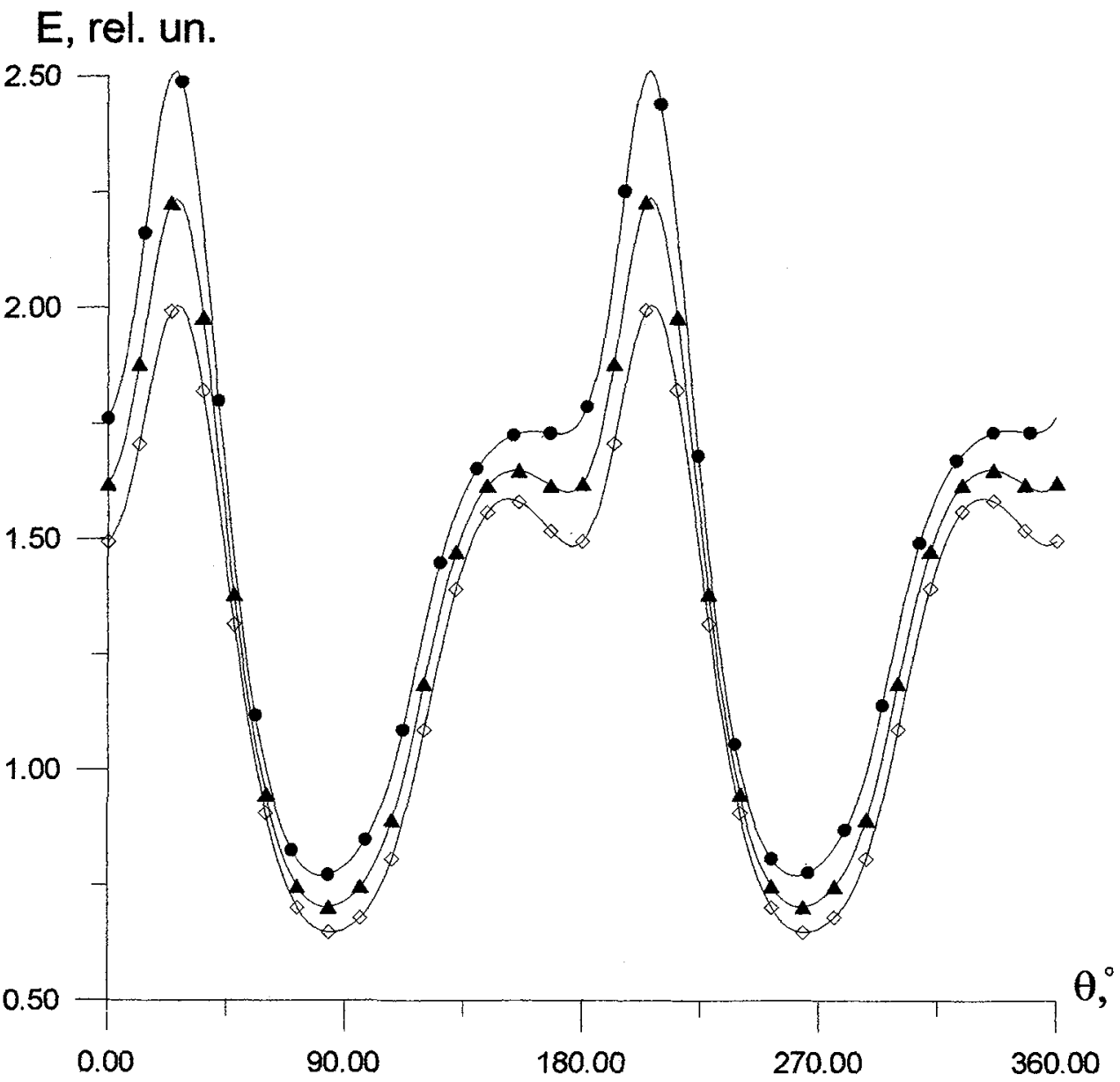

Figure 2. The angle dependence of elastic energy of the DNC for different values of the anisotropy factor: $A=2.0(\diamond), A=1.7(\Delta)$ and $A=1.3(\diamond)$.

Thus, the thermally formed martensite and the stress-induced one correspond to the different combinations of elastic moduli (8) and (9) but, at the same time, they are originated by one DNC. The orientations of vectors $\mathbf{N}$ and $\mathbf{S}$ gained by calculations for the angles $\theta$ close to $171^{\circ}$ and $185^{\circ}$ are presented in the Table 1. Evidently, they correlate well with the above-presented experimental data. 
Table 1. The culculated orientations of normals of habit planes $(\mathbf{N})$ and vectors of macroshear $(\mathbf{S})$ for few values of angle $\theta$.

\begin{tabular}{|c|c|c|c|c|c|c|}
\hline $\boldsymbol{\theta}$ & $170^{\circ}$ & $171^{\circ}$ & $172^{\circ}$ & $184^{\circ}$ & $185^{\circ}$ & $186^{\circ}$ \\
\hline \multirow{3}{*}{$\mathbf{N}$} & 0.8720 & 0.8680 & 0.8636 & 0.7895 & 0.7815 & 0.7734 \\
& 0.4229 & 0.4205 & 0.4178 & 0.3758 & 0.3715 & 0.3670 \\
& 0.2465 & 0.2644 & 0.2823 & 0.4853 & 0.5012 & 0.5169 \\
\hline \multirow{3}{*}{$\mathbf{S}$} & 0.506 & 0.5149 & 0.5237 & 0.6272 & 0.6357 & 0.6440 \\
& -0.4905 & -0.4803 & -0.4699 & -0.3401 & -0.3296 & -0.3193 \\
& -0.7095 & -0.7101 & -0.7106 & -0.7006 & -0.6981 & -0.6952 \\
\hline
\end{tabular}

\section{CONCLUSION}

Successful identification of dislocation-based nucleation centers of martensite and experiments on the simulation of initial excited state $[3,10]$ testify that the mechanism of dislocation-based nucleation and wave growth are abundant. Macroscopic morphological attributes gain the natural treatment; in so doing to invoke data about the final phase is not necessary. It must be emphasized that the controlling wave process transfers the threshold deformation only. It provides the co-operativeness of atom displacements at the mesoscale and doesn't predetermine the ultimate deformation and short-range atom displacements. It is the existence of controlling process that is the specificity of martensitic transformation and therefore the comprehension of content of controlling mechanism is tantamount to the comprehension of the physical nature of martensitic transformations.

\section{References}

[1] Wayman C.M. Diffusionless Phase Transformations, in: Physical Metallurgy, 3th Ed., R.W.Cahn and P.Haasen Eds. (North-Holland Amsterdam, 1983), Ch.15.

[2] Kashchenko M.P., The wave model of martensite growth for $\gamma-\alpha$ transformation in iron-based alloys (UIF Nauka, Ekaterinburg, 1993) 224p.

[3] Kashchenko M.P., Letuchev V.V., Konovalov S.V. and Neskoromnyi S.V., Phys. Met. Metallogr. 76(1993) 300-308.

[4] Olson G.B. and Roitburd A.L. Martensite Nucleation, in:Martensite, G.B.Olson and W.S.Owen Eds. (ASM Int. The Materials Information Society, 1992), 149-174.

[5] Kashchenko M.P., Vereshchagin V.P. and Aristova N.V., Phys. Met. Metallogr. 75(1993) 135-138.

[6] Alexina I.V., Aristova N.V., Letuchev V.V. and Kashchenko M.P., "The dislocation nucleation centers at B2-B19 martensitic transformation in Ti-Ni-Cu alloys", Shape memory materials, Beijing 25-28 September 1994, Chu Youyi and Tu Hailing Eds. (International Academic Publishers, Beijing, 1994) pp.65-68.

[7] Wayman C.M., "Phase transformations in Ni-Ti - type shape memory alloys", The International Conference on Martensitic Transformations, 1986 (The Japan Institue of metals, 1987) pp.645-652.

[8] Mercier.O, Melton K., Greemaud G. and Hagi J., J.Appl.Phys. 51(1980)1833-1834.

[9] Kuznetsov A.V., Muslov S.A., Lotkov A.l., Khachin V.N., Grishkov V.N. and Pushin V.G., Izvestiya Vuzov Fizika 30(1987)98-99.

[10] Letuchev V.V., Konovalov S.V. and Kashchenko M.P., "Dynamical lattice state at the initial stage of martensitic transformation and possibilities of its physical realization", European Symposium on Martensitic Transformation, Barselona 14-16 September 1994, A.Planes, J.Ortin and L.Manosa Eds.(Les Edition de Physique, Les Ulis, 1995) pp.53-58. 